
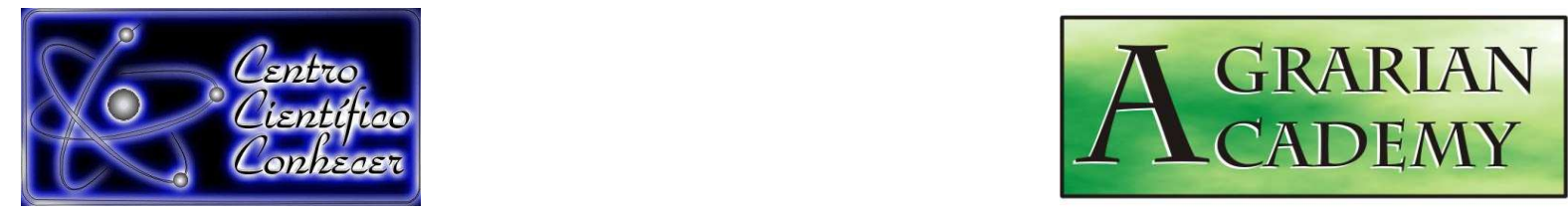

\title{
VALORAÇÃO ECONÔMICA DO MEL NO ESTADO DE GOIÁS: CONSERVAÇÃO E RENDA
}

Fernanda Duarte Araújo1, Carlos de Melo e Silva-Neto ${ }^{1 *}$, Anna Clara Chaves Ribeiro², Guilherme Murilo Oliveira ${ }^{1}$, Abadia dos Reis Nascimento ${ }^{1}$

1 Escola de Agronomia, Universidade Federal de Goiás, Goiânia. 2 Instituto de Ciências Biológicas, Universidade Federal de Goiás, Goiânia *carloskoa@gmail.com

Recebido em: 16/11/2015 - Aprovado em: 10/12/2015 - Publicado em: 21/12/2015 DOI: http://dx.doi.org/10.18677/Agrarian_Academy_022

\section{RESUMO}

Estado de Goiás é pouco expressivo no cenário nacional de produção apícola, mesmo apresentando clima, relevo e vegetação adequados para a atividade. Porém nos últimos anos, a produção de mel vem crescendo, surgindo como alternativa para a diversificação de renda e até mesmo atividade principal nas propriedades rural. Assim o objetivo deste trabalho consistiu em caracterizar o cenário econômico e a dinâmica da produção de mel no Estado de Goiás. As produções anuais de mel no Estado de Goiás dos períodos de 1998 a 2013 foram obtidas por meio dos dados do Instituto Mauro Borges de Estatística. Os preços do mel (por tonelada) nos diferentes anos foram obtidos a partir de diferentes de valores definidos pela Câmara setorial de Mel e produtos apícolas. A produção de mel no Estado de Goiás gerou nos últimos 16 anos $(1998$ - 2013) cerca de $R \$ 21$ milhões de reais. Anualmente a produção de mel movimenta $R \$ 2$ milhões de reais somente com 0 valor do produto, sem contar com outros componentes da cadeia produtiva do mel. O preço do quilo do mel aumentou mais de $207 \%$ no Estado de Goiás entre os anos de 1998 a 2013, já a produção de mel aumentou cerca de 308\%. O valor pago cresceu nos últimos 16 anos no Estado de Goiás. A produção de mel no Estado de Goiás deverá continuar a crescer nos próximos anos, porém o valor pago ao produto deverá ter seu preço estabilizado.

PALAVRAS-CHAVE: Apicultura, Orizona, produção de mel, produção familiar.

\section{HONEY ECONOMIC VALUATION IN THE STATE OF GOIÁS: CONSERVATION AND INCOME}

\begin{abstract}
The Goiás State is not prominent on the national scene of beekeeping, even with climate, topography and vegetation appropriate for the activity. But in recent years honey production has been growing, emerging as an alternative for the diversification of income and even main activity in rural properties. So the aim of this work is characterized the economic environment and the dynamics of honey production in the state of Goiás. The annual productions of honey in the Goiás State on the periods from 1998 to 2013 were obtained using data from the Institute of Statistics Mauro Borges. Honey prices (per tonne) in different years were obtained from
\end{abstract}


different values defined by Sectoral House of Honey and Bee products. Honey production in the Goiás generated in the last 16 years (1998-2013) about R \$21 million. Every year honey production moves $R \$ 2$ million reais only with the value of the product, not counting other components of the productive chain of honey. The price of a kilogram of honey has increased by over $207 \%$ in the Goiás between the years 1998 to 2013 have honey production increased about 308\%. The value of honey increased in the last 16 years in the Goiás. The production of honey in Goiás State should continue to grow in coming years, but the amount paid to the product should have its stabilized price.

KEYWORDS: beekeeping, honey production, family farms, Orizona.

\section{INTRODUÇÃO}

A criação de abelhas é uma atividade tradicional associada à história da humanidade. Desde 2.400 anos a.C., os egípcios começaram a colocar as abelhas em potes de barro. Assim, o homem começa a criar e transportar as abelhas no intuito de manter disponível a produção de mel para consumo.

A criação de Apis mellifera L., ou apicultura, surgiu no Brasil em torno de 1840, com abelhas oriundas da Espanha e Portugal, trazidas pelo Padre Antônio Carneiro. Já em meados de 1950 houve a introdução das abelhas africanizadas, que mesmo com controvérsias científicas, melhorou a qualidade das abelhas, permitindo a expansão e crescimento da atividade no país (PEREIRA et al., 2003).

A produção de mel do Brasil é destaque no cenário mundial, estando entre os países em que a produção apícola mais cresce atualmente. Em 2013, o país produziu cerca de 35.364 toneladas de mel, ficando entre os 15 maiores produtores de mel. Dentro do país, a região Sul possui $49 \%$ da produção de mel do país. Já Nordeste e Sudeste, representam 18\% e 17\% respectivamente da produção de mel. Assim, dentre todos os estados brasileiros, somente nove estados juntos são responsáveis por mais de 84\% da produção do mel nacional (SEBRAE, 2014).

O Estado de Goiás não se destaca no cenário nacional de produção apícola, mesmo apresentando clima, relevo e vegetação adequados para a atividade. Nos últimos anos, a produção de mel vem crescendo, surgindo como alternativa para a diversificação de renda e até mesmo atividade principal nas propriedades rural (GOIÁS, 2015).

Além do crescimento, a atividade apícola é vista como atividade econômica com características socioambientais, uma vez que, gera renda aos produtores rurais e permite a ação das abelhas nos serviços ecossistêmicos, gerando ganhos indiretos com polinização de culturas agrícolas e plantas nativas (BIZOTTO \& SANTOS, 2015; GIANNINI et al., 2015). Desta forma, objetivou-se caracterizar o cenário econômico e a dinâmica da produção de mel no Estado de Goiás.

\section{MATERIAL E MÉTODOS}

\section{Produção e valor do mel}

As produções anuais de mel (PROD) no Estado de Goiás nos períodos de 1998 a 2013 foram obtidas por meio dos dados do Instituto Mauro Borges de Estatística (2015). Os preços do mel (por tonelada) nos diferentes anos foram obtidos a partir de diferentes de valores definidos pela Câmara Setorial de Mel e produtos apícolas (CSMA, 2015). Para definição dos valores totais foram utilizadas as seguintes equações - adaptadas de GALLAI \& VAISSIÈRE (2009): 


$$
\begin{gathered}
\text { Vtotal } i=\underset{\text { Vano } 1}{\text { Vano }=\text { PRODo } 2 *}+\ldots \text { Vano } i \\
\text { PROD }
\end{gathered}
$$

Vtotal $i$ - Valor do mel por todo o período avaliado.

Vano - Valor do mel no ano.

PROD - Produção do mel por ano em tonelada (ton).

$\mathrm{P}$ - Preço da tonelada do mel no ano ( $\mathrm{R} \$$ - Reais).

Para evidenciar a relação entre quantidade de mel produzida e o valor do mel no ano foi realizado regressão quadrática com nível de significância de $99 \%$. Para verificar o ajuste da equação aos dados foram observados o coeficiente de regressão $\left(R^{2}\right)$ e a distribuição dos resíduos.

\section{RESULTADOS E DISCUSSÃO}

A produção de mel no Estado de Goiás gerou nos últimos 16 anos (1998 2013) cerca de $R \$ 21.300 .611,68$ de reais. Anualmente a produção de mel movimenta $R \$ 2.505 .954,31$ de reais somente com o valor do produto, sem contar com outros componentes da cadeia produtiva do mel como própolis, colmeias e etc. No intervalo de 1998 a 2013, os valores arrecadados com a produção de mel aumentaram quase sete vezes, devido principalmente ao aumento na produção do mel e na valorização do produto no mercado (Figura 01).

O preço do quilo do mel aumentou em cerca de $207 \%$ no Estado de Goiás entre os anos de 1998 a 2013, já a produção de mel aumentou cerca de $308 \%$ no mesmo período. A escalada da produção do mel ocorreu fortemente no ano de 2005 , quando a produção do mel aumentou em $125 \%$, impulsionado pelo aumento do preço do produto que quase dobrou (devido à alta do valor do dólar em relação ao real) entre os anos de 2002 para 2003, estimulando assim o investimento em novas colmeias, melhoria e incremento da produção apícola no Estado.

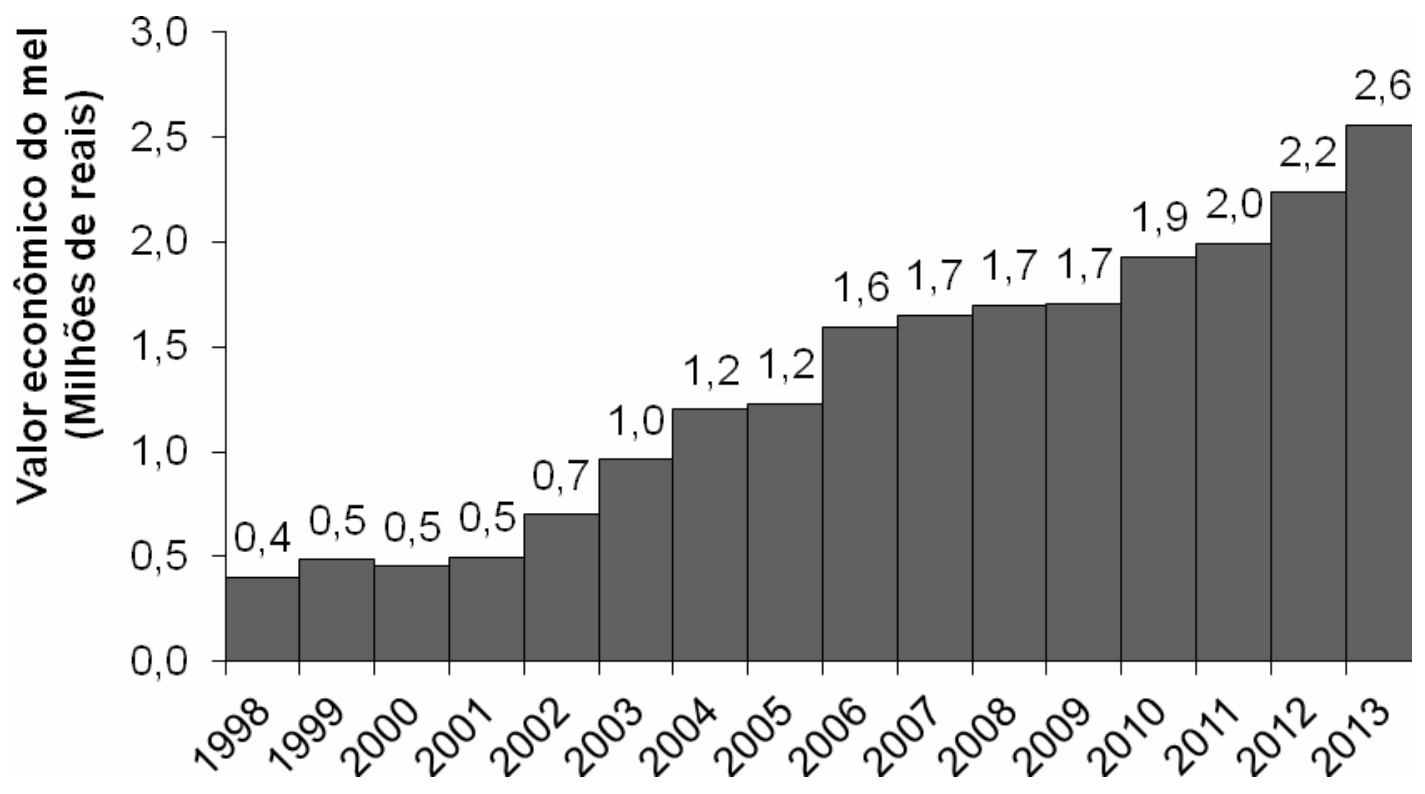

FIGURA 01. Valor econômico do mel produzido no Estado de Goiás nos anos de 1998 a 2013. 
O Estado de Goiás não apresenta produção apícola expressiva no cenário nacional, porém o crescimento da produção é considerável e pode se destacar entre os outros Estados futuramente. Dentro de Goiás, os municípios que são destaques históricos na produção apícola correspondem à: Orizona, Silvânia, Porangatu, Goiandira, Vianópolis, Pontalina, Cidade de Goiás, Heitoraí, Uruana e Chapadão do Céu, sendo estes os maiores produtores nos últimos 16 anos, em ordem decrescente (Figura 02).

Já em relação à produção média anual, outros municípios também são destaque na produção: Orizona, Jataí (com aumento nos últimos anos), Silvânia, Porangatu, Goiandira, Vianólopis, Niquelândia (também com aumento nos últimos anos), Pontalina, Cidade de Goiás e Heitoraí, também em ordem decrescente de produção média de mel por ano. Os dez municípios que mais produzem mel no Estado concentram cerca de $44,6 \%$ da produção estadual, refletindo assim que a produção é concentrada em poucos locais (Figura 03).

A relação entre produção de mel no Estado de Goiás e o valor pago ao produtor por quilo também apresentou significância $(p<0,000)$, sendo verificada relação positiva entre 0 valor pago e a produção de mel $\left(R^{2}=0,79 ; \quad y=-\right.$ $\left.19,64 x^{2}+282,1 x-677\right)$ (Figura 04). No cenário inicial de produção de mel e atividade apícola, o valor pago ao produtor pelo mel é decisivo como incentivo para o aumento da produção. Mesmo assim, o aumento nos preços deverá se manter estável entre $\mathrm{R} \$ 6,00$ e $\mathrm{R} \$ 7,50$, porém de acordo com o volume de mel produzido nos próximos anos, o valor poderá reduzir devido o aumentando da oferta de produto, reduzindo os valores pagos aos produtores.

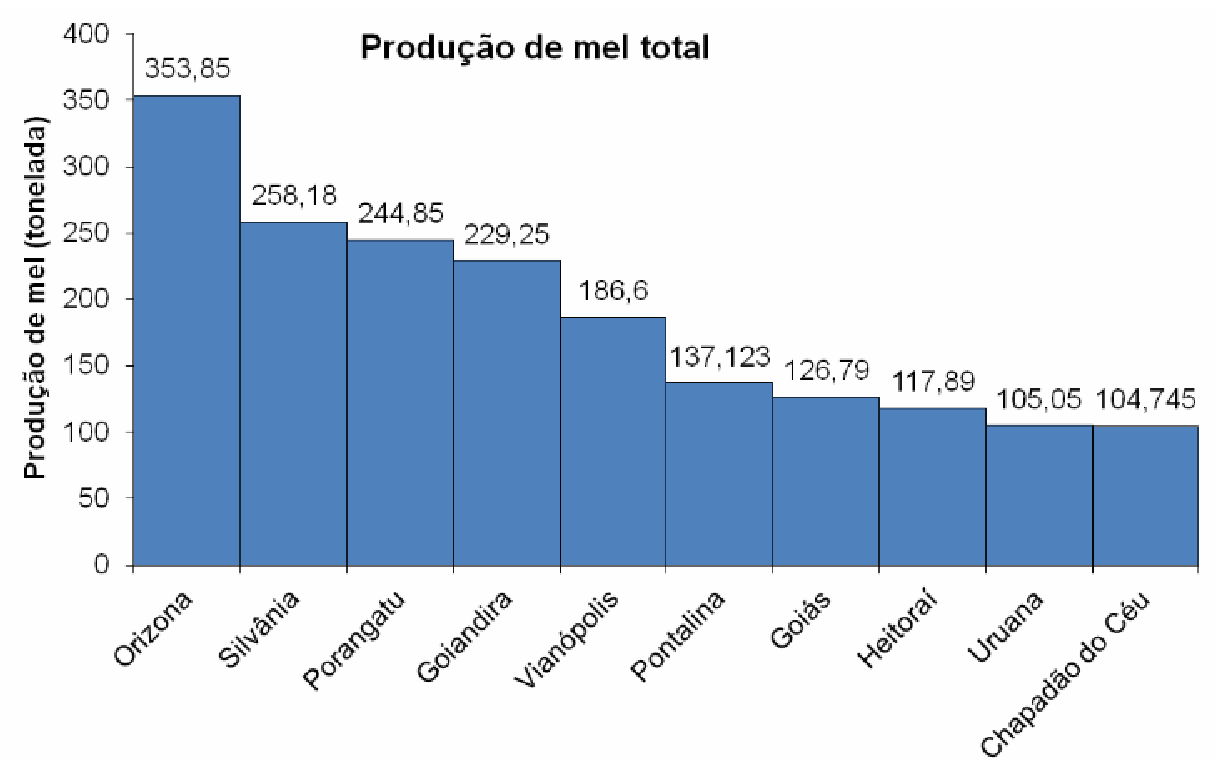

FIGURA 02. Produção total de mel no período de 1998 a 2013 de mel por município do Estado de Goiás. 


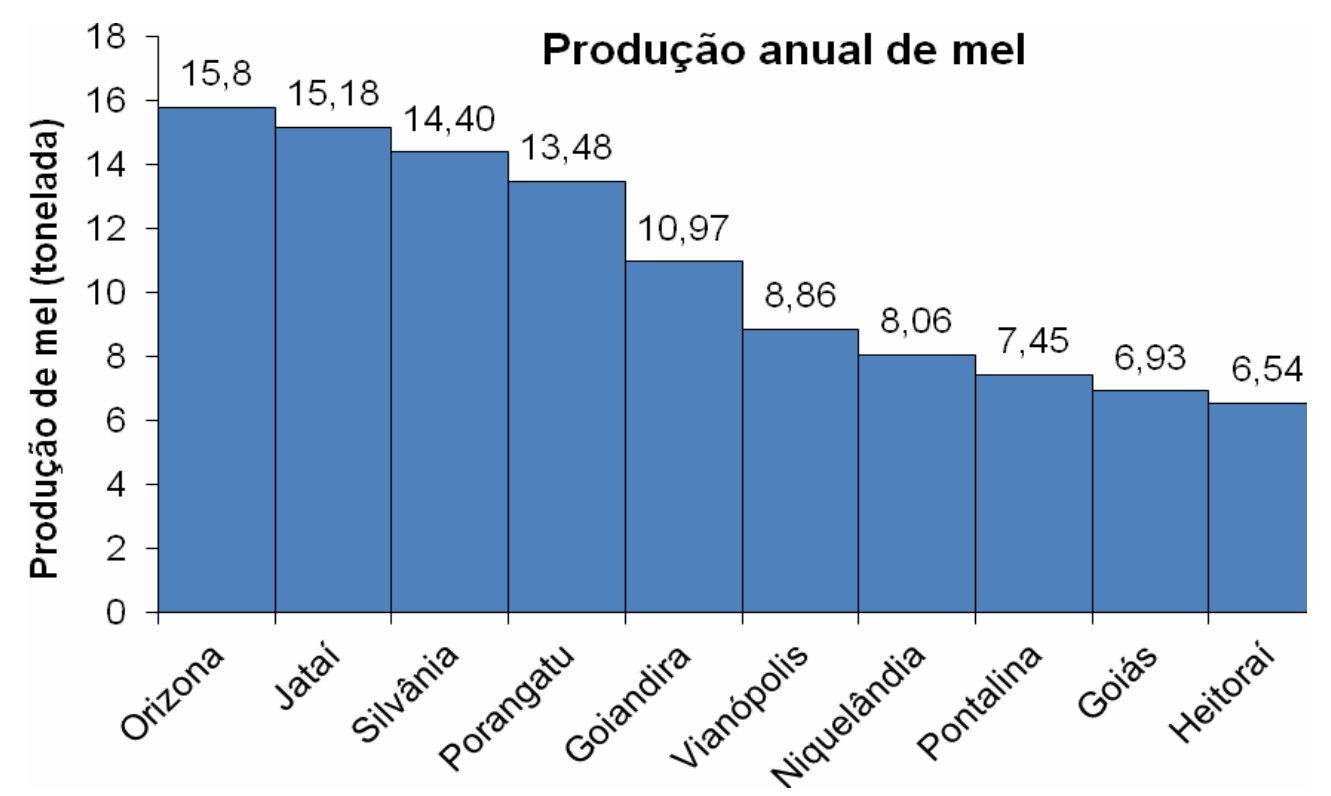

FIGURA 03. Produção média anual (1998-2013) de mel por município do Estado de Goiás.

Além do investimento local e organização dos produtores para a produção do mel, é relevante destacar a aptidão apícola de cada região. O uso da terra e atividades no entorno dos locais de instalação do apiário são fatores que podem comprometer a quantidade e qualidade do mel produzido (WOLFF et al., 2006; MOURA et al., 2014; DAMASIA-GOMES et al., 2015). Dentre os municípios goianos, muitos apresentam intensa atividade agrícola com culturas temporárias e alto impacto sobre os recursos naturais, sendo que poucos apresentam atividades sustentáveis alternativas com menor impacto sobre os recursos naturais.

A apicultura, dentre as atividades agrícola, é considerada alternativa sustentável para a geração de renda e utilização dos recursos naturais de forma não convencional, uma vez que pode utilizar-se de espaços alternativos. Além disso, possibilita 0 aproveitamento de vegetação nativa como recurso alimentar e para instalação das colônias, não utilizando-se de áreas consideradas produtivas para culturas como soja, milho, feijão e cana-de-açúcar (ALMEIDA \& CARVALHO, 2009; KHAN et al., 2009).

Outro aspecto relevante é o inicio da criação de abelhas para os produtores rurais. No início, há uma percepção que a atividade é secundária. No entanto, com os investimentos tecnológicos adequados, organização para produção e comercialização do mel e assistência técnica, a apicultura se torna a atividade principal na geração de renda de muitas propriedades (BOTH et al., 2009; BARBOSA \& SOUZA, 2013;OLIVEIRA et al., 2013). 


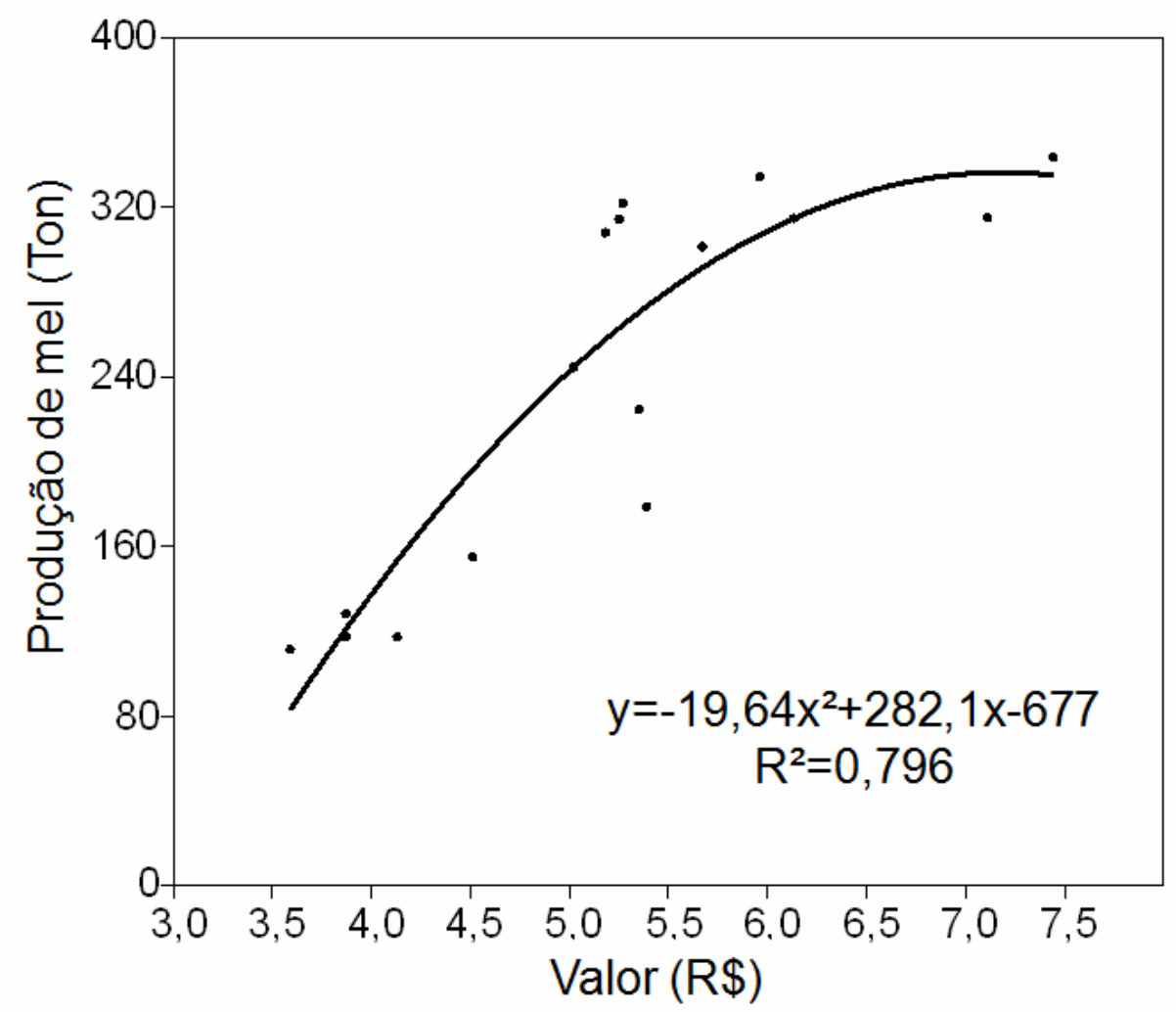

FIGURA 04. Relação entre produção de mel (reais) e valor pago (por quilo) no Estado de Goiás (y: produção de mel em tonelada; x: valor pago pelo quilo do mel; $y=-19,64 x^{2}+282,1 x-677 ; R^{2}=0,796 ; F=25,50$; $p=0,000)$.

Os principais pontos relacionados aos benefícios ambientais da atividade apícola são: atividade de baixo impacto ambiental; a ação de polinização das abelhas aumenta a produtividade de diversas lavouras e culturas além das plantas nativas (RIBEIRO et al., 2015); favorece a preservação da biodiversidade, uma vez que as plantas da área estão sendo polinizadas e trocando material genético, aumentando sua variabilidade (MELO-SILVA et al., 2013); auxiliam na recuperação de áreas degradadas, pois as espécies polinizadas produzirão mais sementes, contribuindo para a regeneração natural (MORANDIN \& KREMEN, 2013; SORDI \& SHLINDWEIN, 2014).

A atividade apícola também auxilia na disseminação da consciência ambiental entre os produtores e população, especialmente em questões como conservação de fragmentos florestais, que fornecem alimento e abrigo para as abelhas e no uso de agrotóxicos, aos quais as abelhas são muito sensíveis (GALLAI \& VAISSIÈRE, 2009; FAO, 2013; SORDI \& SHLINDWEIN, 2014).

Já os principais pontos relacionados aos benefícios sociais da atividade apícola correspondem à geração de renda e ocupação aos produtores, resultando assim na manutenção do homem no campo e a diminuição do êxodo rural, além da criação de empregos por toda a cadeia produtiva apícola, com perspectivas de desenvolvimento de associações, cooperativas e demais organizações sociais, 0 que favorece a distribuição equitativa dos ganhos com a atividade (SORDI \& SHLINDWEIN, 2014). Outro aspecto importante para a apicultura é que a produção familiar corresponde a grande parte dos criadores de abelhas em todo o Brasil, 
sendo a atividade adequada para o sistema familiar (BARBOSA \& SOUSA, 2013; OLIVEIRA et al., 2013; BOTH \& BOTH, 2009). Porém, a produção apícola com menor número de trabalhadores limita o número de colmeias a serem manejadas e assim a produção de mel, sendo o número ideal entre 100 e 200 colmeias (SABBAG \& NICODEMO, 2011).

Segundo FREITAS et al. (2004), os apicultores capacitados apresentam maior produtividade das colmeias em comparação com os não capacitados. O domínio das técnicas de manejo apícolas, dos enxames e a manutenção adequada refletem na produção das safras até mesmo em longo prazo. Dentre as estratégias para valorização da cadeia produtiva apícola está a diversificação dos produtos, aproveitando as diversas possibilidades produzidas na colmeia, como própolis, pólen, abelhas, colônias, favos e o próprio mel. Outro aspecto a se considerar é a produção orgânica com certificações relativas a conformidade de qualidade e ambiental, valorizando ainda mais o produto final.

Assim é essencial que os produtores apícolas de Goiás realizem o manejo adequado dos apiários com assistência técnica qualificada para buscar melhorias técnicas e tecnológicas, aliadas com pesquisas visando ao aumento da qualidade e quantidade da produção. Futuros estudos relacionados a sanidade, manejo de abelhas, colheita e pós-colheita do mel poderão melhorar a produção em Goiás, elevando o papel do Estado no cenário apícola nacional.

\section{CONCLUSÃO}

A produção de mel no Estado de Goiás gerou nos últimos 16 anos mais de 21 milhões de reais com média anual acima de 2,5 milhões de reais. Os principais municípios do Estado de Goiás em ganhos econômicos com o mel são: Orizona, Silvânia, Porangatu e Goiandira.

O valor pago ao mel cresceu nos últimos 16 anos no Estado de Goiás. A produção de mel deverá continuar a crescer nos próximos anos, porém o valor pago ao produto deverá ter seu preço estabilizado.

\section{REFERÊNCIAS}

ALMEIRA, M. A. D.; CARVALHO, C. M. S. Apicultura: uma oportunidade de negócio sustentável. Salvador: Sebrae Bahia, 2009. 52 p. Disponível em: <http://hotsites.diariodepernambuco.com.br/economia/2012/sertaodoce/docs/apicultu ra-sustentavel.pdf $>$.

BARBOSA, W. D. F.; SOUSA, E. P. D. Nível tecnológico e seus determinantes na apicultura cearense. Revista de Política Agrícola, v. 22, n. 3, p. 32-47, 2013. Disponível em: <http://ainfo.cnptia.embrapa.br/digital/bitstream/item/92887/1/Niveltecnologico-e-seus-determinantes-na-apicultura-cearense.pdf $>$.

BIZOTTO, L. A.; SANTOS, R. S. Dinâmica de voo e coleta de recursos por Apis mellifera em pomar de macieira. Enciclopédia Biosfera, v.11, n. 21, p. 3499, 2015. Disponível em: <http://www.conhecer.org.br/enciclop/2015b/biologicas/Dinamica\%20de\%20voo\%20 Apis.pdf>.

BOTH, J.; KATO, O. R.; OLIVEIRA, T. F. Perfil socioeconômico e tecnológico da apicultura no município de Capitão Poço, estado do Pará, brasil. Amazônia: Ciência 
\& Desenvolvimento, v. 5, n. 9, p. s/n, 2009. Disponível em: $<$ https://www.embrapa.br/busca-de-publicacoes/-/publicacao/783287/perfilsocioeconomico-e-tecnologico-da-apicultura-no-municipio-de-capitao-poco-estadodo-para-brasil>.

BOTH, J. P. C. L.; BOTH, A. L. C. M. Mel na Composição da Renda em Unidades de Produção Familiar no Município de Capitão Poço, Pará, Brasil. Cadernos de Agroecologia, v. 4, n. 1, p. 931-934, 2009. Disponível em: <https://www.embrapa.br/busca-de-publicacoes/-/publicacao/661406/mel-nacomposicao-da-renda-em-unidades-de-producao-familiar-no-municipio-de-capitaopoco-para-brasil>.

CORONEL, D. A.; SOUSA, E. P.; AMORIM, A. L. Desempenho exportador do mel natural nos estados brasileiros. Pesquisa \& Debate. Revista do Programa de Estudos Pós-Graduados em Economia Política, v. 22, n. 2, p. 343-360, 2011. Disponível em: http://revistas.pucsp.br/index.php/rpe/article/view/11749

CSMA, 2015. Câmara Setorial de Mel e produtos apícolas. Ministério de Agricultura e Pecuária. Disponível em: $<$ http://www.agricultura.gov.br/arq_editor/file/camaras_setoriais/Mel_e_produtos_api colas/36RO/ICA_36RO.pdf. Novembro de 2015>.

DAMASIA-GOMES, L.; FALEIRO, K. M.; SANTOS, S. O.; GUIMARAES, L. E.; SILVA-NETO, C. M. Physical-chemical characteristics of honey on Brazil. Enciclopédia Biosfera, v. 11, n. 22, p. 670-682, 2015. Disponível em: <http://dx.doi.org/10.18677/Enciclopedia_Biosfera_2015_110>. DOI: 10.18677/Enciclopedia_Biosfera_2015_110

FREITAS, D. G. F.; KHAN, A. S.; SILVA, L. M. R. Nível Tecnológico e Rentabilidade de Produção de Mel de Abelha (Apis mellifera) no Ceará. Revista de Economia e Sociologia Rural, v. 42, n. 1, p.171-188, 2004. Disponível em: <http://dx.doi.org/10.1590/S0103-20032004000100009>. DOI: $10.1590 / S 0103-$ 20032004000100009

GALLAI, N.; VAISSIÈRE, B. Guidelines for the economic valuation of pollination services at a national scale, Food and Agriculture Organization of the United Nations, p. 20, Rome, Italy, 2009. Disponível em: <http://www.fao.org/3/aat523e.pdf $>$.

GIANNINI, T. C.; CORDEIRO, G. D.; FREITAS, B. M.; SARAIVA, A. M.; IMPERATRIZ-FONSECA, V. L. The Dependence of Crops for Pollinators and the Economic Value of Pollination in Brazil. Journal of Economic Entomology, v. 93, s/n, s/p, 2015. Disponível em:

<http://dx.doi.org/10.1093/jee/tov093>. DOI: 10.1093/jee/tov093

GOIÁS. Instituto Mauro Borges de Estatística E Estudos Socioeconômicos. Produção agrícola do Estado de Goiás. Disponível em: <http://www.seplan.go.gov.br/sepin/perfilweb/Estatistica_bde.asp>. 
KHAN, A. S.; MATOS, V. D.; LIMA, P. V. P. S. Desempenho da apicultura no estado do Ceará: competitividade, nível tecnológico e fatores condicionantes. Revista de Economia e Sociologia Rural, v.47, n. 3, p. 651-676, 2009. Disponível em: <http://dx.doi.org/10.1590/S0103-20032009000300006>. DOI: 10.1590/S010320032009000300006

MELO-SILVA, C.; FRANCESCHINELLI, E. V. ; STEHMANN, J. R.; MESQUITANETO, J. N. Preliminary study on genetic diversity of endemic and threatened species of Petunia (Solanaceae). Darwiniana, v. 1, n. 2, p. 220-226, 2013. Disponível <http://www.ojs.darwin.edu.ar/index.php/darwiniana/article/view/526/567>.

em:

MORANDIN, L. A.; KREMEN, C. Bee preference for native versus exotic plants in restored agricultural hedgerows. Restoration Ecology, v. 21, n. 1, p. 26-32, 2013. Disponível em: <http://dx.doi.org/10.1111/j.1526-100X.2012.00876.x>.

DOI: $10.1111 / \mathrm{j} .1526-100 X .2012 .00876 . x$

MOURA, S. G.; MURATORI, M. C. S.; MONTE, A. M.; CARNEIRO, R. M.; SOUZA, D. C.; MOURA, J. Z. Qualidade do mel de Apis mellifera L. relacionadas às boas práticas apícolas. Revista Brasileira de Saúde e Produção Animal, v. 15, n. 3, p. 731-739, 2014. Disponível em: < http://dx.doi.org/10.1590/S151999402014000300021>. DOI: 10.1590/S1519-99402014000300021

OLIVEIRA, O. V.; OLIVEIRA, F. C.; PESSOA, R. A. Benefícios dos arranjos produtivos locais: A percepção dos apicultores sobre a rede abelha Ceará - Brasil. Redes, v. 18, n. 3, p. 118-137, 2013. Disponível em: <http://dx.doi.org/10.17058/redes.v18i3.1610>. DOI: 10.17058/redes.v18i3.1610

PEREIRA, F. D. M.; LOPES, M. D. R.; CAMARGO, R. D.; VILELA, S. D. O. Produção de mel. Embrapa Meio-Norte, v. 3, s/n, 2003. Disponível em: <http://www.cpamn.embrapa.br/publicacoes/documentos/2003/doc_81.pdf>.

RIBEIRO, A. C. C. ; SILVA-NETO, C. M.; GONÇALVES, B. B.; MESQUITA-NETO, J. N.; MELO, A. P. C. ; BUZIN, E. J. W. K. Economic value of bee pollination in crop production in the State of Goiás. Enciclopédia Biosfera, v. 11, n. 22, p. 3592-3603, 2015. Disponível em: <http://dx.doi.org/10.18677/Enciclopedia_Biosfera_2015_256>. DOI: 10.18677/Enciclopedia_Biosfera_2015_256

SABBAG, O. J.; NICODEMO, D. Viabilidade econômica para produção de mel em propriedade familiar. Pesquisa Agropecuária Tropical, v. 41, n. 1, p. 94-101, 2011. Acesso em: <http://www.scielo.br/pdf/pat/v41n1/a08v41n1.pdf>.

SEBRAE, 2014. Comercialização do mel brasileiro para produção, exportação e importação. Apicultura, Boletim de Tendências. Disponível em: $<$ https://atendimento.sebrae-sc.com.br/webroot/projetos/portal_sebraesc/downloads/mercado/2014/boletins/boletim-janeiro-2014-apicultura.pdf>.

SORDI, V. F.; SHLINDWEIN, M. M. Os principais benefícios da atividade apícola e os entraves para seu desenvolvimento no estado de Mato Grosso do Sul. Revista 
em Agronegócios e Meio Ambiente, v. 7, n. 3, p. 571-590, 2014. Disponível em: <http://periodicos.unicesumar.edu.br/index.php/rama/article/viewFile/2978/2401>.

WOLFF, L. F.; LOPES, M. D. R.; PEREIRA, F. D. M.; CAMARGO, R. C. R.; NETO, J. M. V. Localização do apiário e instalação das colméias. Embrapa Meio-Norte, s/n, $2006 . \quad$ Disponível em: <http://www.cpamn.embrapa.br/publicacoes/documentos/2006/doc_151.pdf>. 